\title{
Relationship of screen-based symptoms for mild traumatic brain injury and mental health problems in Iraq and Afghanistan veterans: Distinct or overlapping symptoms?
}

\author{
Shira Maguen, PhD; ${ }^{1-2 *}$ Karen M. Lau, MA; ${ }^{1}$ Erin Madden, MPH; ${ }^{1}$ Karen Seal, MD, MPH ${ }^{1-2}$ \\ ${ }^{1}$ San Francisco Department of Veterans Affairs (VA) Medical Center, San Francisco, CA; ${ }^{2}$ University of California, \\ San Francisco, San Francisco, CA; and Health Services Research and Development Research Enhancement Award \\ Program, San Francisco VA Medical Center, San Francisco, CA
}

\begin{abstract}
This study used factor analytic techniques to differentiate distinct from overlapping screen-based symptoms of traumatic brain injury (TBI), posttraumatic stress disorder (PTSD), and depression in Iraq and Afghanistan veterans. These symptoms were derived from screen results of 1,549 veterans undergoing Department of Veterans Affairs postdeployment screening between April 2007 and January 2010. Veterans with positive TBI screens were approximately twice as likely to also screen positive for depression and PTSD (adjusted relative risks $=1.9$ and 2.1, respectively). Irritability was a shared symptom between TBI and PTSD, and emotional numbing was a shared symptom between PTSD and depression. Symptoms unique to TBI included dizziness, headaches, memory problems, and light sensitivity. Four separate constructs emerged: TBI, PTSD, depression, and a fourth construct consisting of hypervigilance and sleep problems. These findings illuminate areas of overlap between TBI and common postdeployment mental health problems. Discriminating symptoms of TBI from mental health problems may facilitate diagnosis, triage to specialty care, and targeted symptom management. The emergence of a fourth factor consisting of sleep problems and hypervigilance highlights the need to attend to specific symptoms in the postdeployment screening process.
\end{abstract}

Key words: depression, factor analysis, hypervigilance, insomnia, mental health, OIF/OEF, posttraumatic stress disorder, screen, traumatic brain injury, veteran.

\section{INTRODUCTION}

Traumatic brain injury (TBI) has received greater public attention because of the conflicts in Iraq and Afghanistan (Operation Iraqi Freedom [OIF]/Operation Enduring Freedom [OEF]) [1]. An estimated 22 percent of servicemembers returning from OIF have reported experiencing TBIs and concussions [2], and of injured OIF/OEF servicemembers, 31 percent have been diagnosed with a TBI [3]. Although TBIs range in severity, mild TBI comprises roughly 77 percent of all head injuries

\footnotetext{
Abbreviations: $\mathrm{CFA}=$ confirmatory factor analysis, $\mathrm{CFI}=$ Comparative Fit Index, $d f=$ degree of freedom, EBT = evidencebased treatment, EFA = exploratory factor analysis, $\mathrm{OEF}=$ Operation Enduring Freedom, OIF = Operation Iraqi Freedom, PCS = postconcussive symptom, PTSD = posttraumatic stress disorder, RMSEA = root mean square error of approximation, $\mathrm{TBI}=$ traumatic brain injury, $\mathrm{TBI}+=$ positive screen for TBI, TBI $-=$ negative screen for TBI, TLI $=$ Tucker Lewis Index, VA = Department of Veterans Affairs, WLSMV = weighted least-squares with mean and variance adjustment.

*Address all correspondence to Shira Maguen, PhD; San Francisco VA Medical Center, 4150 Clement St (116-P), San Francisco, CA 94121; 415-221-4810, ext 2511.

Email: Shira.Maguen@va.gov

http://dx.doi.org/10.1682/JRRD.2011.02.0015
} 
among OIF/OEF veterans and is most challenging to diagnose because of symptom overlap with mental health disorders: posttraumatic stress disorder (PTSD) and depression, in particular [4-5].

In an attempt to increase early detection of mild TBI in returning combat veterans entering the Department of Veterans Affairs (VA) system, the VA implemented the first-level TBI screen in April 2007 [6]. The VA considers OIF/OEF veterans to be at risk for having had a possible mild TBI if, on the first-level VA TBI screen, they report a head injury with loss of consciousness or altered mental status and postconcussive symptoms (PCSs, immediately following the exposure and within $1 \mathrm{wk}$ prior to screening) [7]. This definition is sensitive and identifies at-risk OIF/OEF veterans but is not necessarily specific for a TBI diagnosis because mental health conditions, such as PTSD and depression, have also led to positive responses on the VA TBI screen [8]. OIF/OEF veterans who screen positive on the VA first-level TBI screen are therefore referred for second-level TBI screening to conduct a more in-depth assessment for a history of a TBI exposure.

Given that TBI results from exposure to a traumatic event, such as a blast, symptoms of TBI commonly cooccur with both PTSD and depression [9]. TBI can increase the risk of developing PTSD and other mental health conditions because the event associated with the head injury was potentially life-threatening and was associated with other traumatic war zone exposures [10-11]. In recent studies among OIF/OEF veterans with mild TBI, an estimated 33 to 44 percent also met criteria for PTSD and 33 to 62 percent also met criteria for depression [12-14].

Particularly challenging is the fact that many of the cardinal features of mild TBI, such as memory problems, diminished attention and concentration, irritability, and insomnia, are also hallmark symptoms of PTSD and depression $[10,12]$. Schneiderman et al. found that PTSD was one of the factors most strongly associated with TBIrelated PCSs [15]. Indeed, in one published study of OIF servicemembers with mild TBI, after controlling for the effects of PTSD, headaches were the only distinguishing feature associated with loss of consciousness in the battlefield [12]. Nevertheless, a gap exists in identifying other symptoms that differentiate TBI, PTSD, and depression.

The purpose of this study was to address this gap by identifying distinguishing features of each disorder in order to more accurately discriminate between screenbased symptoms of TBI, PTSD, and depression in OIF/ OEF veterans. Because the symptoms of TBI, PTSD, and depression can overlap clinically, problems with triage and appropriate referral for specialty care can ensue, creating delays in diagnosis and treatment [15-17]. Specifically, using factor analytic techniques, we sought to identify symptoms from TBI, PTSD, and depression screens that were overlapping and, in contrast, symptoms that were uniquely associated with a positive TBI screen (and not better accounted for by PTSD or depression). We focused on VA screen results because they represent the level of information that clinicians use to make initial decisions about diagnosis and treatment of their patients. To our knowledge, no study to date has used the VA postdeployment mental health and TBI screens to evaluate symptom discrimination in OIF/OEF veterans. A better understanding of the symptoms that differentiate among TBI, PTSD, and depression in the initial screening stage may facilitate triage, more accurate diagnosis, and early symptom management of OIF/OEF veterans.

\section{METHODS}

\section{Data Source}

We used VA administrative data for OIF/OEF veterans who were screened for TBI at a VA medical center and five affiliated VA community-based outpatient clinics. We extracted responses to the VA TBI screen and the OIF/OEF postdeployment screen for PTSD and depression from the Veterans Health Information Systems and Technology Architecture. We derived demographic and military service information from the national VA OEF/ OIF Roster [18].

\section{Sample Selection}

The study population consisted of 1,713 OIF/OEF veterans who received a first-level TBI screen from April 1, 2007, through January 8, 2010. Of these, we excluded 164 because their screening was completed at another facility and the results were unavailable $(n=86)$, they had previously received a TBI diagnosis $(n=67)$, they had refused screening $(n=10)$, or they had an incomplete screen $(n=1)$. Our final sample included 1,549 veterans. We found no significant demographic and military service characteristic differences between veterans who were included compared with those who were excluded 
from the study sample. For veterans who had completed more than one TBI screen on different dates $(n=127)$, we used only the most recent and complete screen for this study. When examining the prevalence of mental health conditions, we further restricted the sample to veterans who completed a PTSD screen and depression screen $(n=$ 1,400 and 1,407, respectively) within 1 year of their most recent TBI screen.

Next, in order to perform the more in-depth statistical analyses described later, we selected a subsample of veterans who had completed all four questions of the TBI screen (see "Measures" section for TBI screen structure) and who had completed both a PTSD and depression screen within 1 year of their TBI screen $(n=358)$.

\section{Measures}

\section{Traumatic Brain Injury Screen}

The VA TBI screen is a five-section assessment tool modified from the Brief TBI Screen [19] to evaluate possible TBI in OIF/OEF veterans [20]. The first section of the TBI screen identifies veterans who were previously diagnosed with TBI. Veterans with prior TBI diagnoses were not administered the remainder of the TBI screen. Veterans without a prior TBI diagnosis were subsequently asked four sets of questions [7]. A positive screen for TBI (TBI+) consisted of endorsing one or more questions in each of the four sections: (1) a qualifying TBI event (blast or explosion, vehicular accident and/ or crash, fragment wound or bullet wound above the shoulders, or fall), (2) immediate symptoms following the event (losing consciousness/knocked out; being dazed, confused, or seeing stars; not remembering the event; concussion; or head injury), (3) new or worsening TBI-related symptoms following the event (memory problems or lapses, balance problems or dizziness, light sensitivity, irritability, headaches, or sleep problems), and (4) current symptoms (same as in section 3). A negative screen for TBI (TBI-) occurred when a veteran failed to endorse at least one item in any of the four sections. Once a veteran failed to endorse any items in a particular section of the VA TBI screen, the screen was terminated.

\section{Posttraumatic Stress Disorder Screen}

The Primary Care Posttraumatic Stress Disorder Screen is a brief four-item screen designed to detect possible PTSD symptoms in primary care and other medical settings [21-22]. The screen yields binary responses (“yes" or "no") for each of four PTSD symptom clusters: re-experiencing, avoidance, emotional numbing, and arousal. Endorsing three or more symptoms constituted a positive screen for PTSD [23].

\section{Depression Screen}

The Patient Health Questionnaire-2 [24] is a twoitem depression screen adapted from the nine-item depression module of the Patient Health Questionnaire [25]. This shorter screen assessed symptoms of depressed mood (down and/or hopeless) and anhedonia (little interest) on a four-point Likert scale ranging from 0 ("not at all”) to 3 ("nearly every day"). Total scores range from $0-6$. Veterans who obtained a total score of $\geq 3$ screened positive for depression [24].

\section{Statistical Analysis}

We first compared characteristics of TBI+ and TBIveterans using $t$-tests for continuous variables and Pearson chi-square tests for categorical variables. To compare PTSD and depression screens between TBI+ and TBIveterans, we used log-binomial regression to estimate risk ratios and associated confidence intervals. We adjusted models for potential confounders, including sociodemographics and military factors. We conducted all analyses, other than factor analyses, using SAS version 9.2 (SAS Institute Inc; Cary, North Carolina).

\section{Exploratory Factor Analysis}

We conducted an exploratory factor analysis (EFA) in Mplus, version 5 (Muthén \& Muthén; Los Angles, California) in order to identify a viable factor structure of the 12 different symptoms included in the three screens: TBI screen (memory problems, balance problems or dizziness, light sensitivity, irritability, headaches, and sleep problems), PTSD screen (nightmares, avoidance, emotional numbing, and hypervigilance), and depression screen (little interest and down and/or hopeless). We used a robust weighted least-squares with mean and variance adjustment (WLSMV) method estimator and treated all 12 items as binary or ordinal variables. The WLSMV method estimator provided weighted least-square parameter estimates using a diagonal weight matrix and robust standard errors and a mean- and variance-adjusted chisquare test statistic. We considered the maximum number of factors (7) that were technically possible. We rotated the raw factor loadings using oblique (promax) rotation. We based model selection on consideration of eigenvalues, 
which were the estimated residual variances, as well as the a priori hypothesis that the factor structure would reflect the clinical screens from which the items originated. We retained items with primary factor loadings of $\geq 0.4$.

\section{Confirmatory Factor Analysis}

We then conducted a confirmatory factor analysis (CFA) to confirm the exploratory factor model and to assess whether the factor structure required modification. We used Mplus to estimate the confirmatory factor model, using the WLSMV method estimator. We used the derivatives for parameter estimates as modification indices in order to determine which parameters were most likely to improve model fit. We compared nested models using WLSMV estimates of chi-square and the derivatives difference test for change in model fit, which is a robust goodness-of-fit test where the chi-square is adjusted to obtain an accurate $p$-value [26]. We used the following goodness-of-fit indices to assess the degree of fit between the model and the data: Tucker Lewis Index (TLI; acceptable: >0.90; excellent: >0.95), Comparative Fit Index (CFI; acceptable: >0.90; excellent: >0.95) [27], and root mean square error of approximation (RMSEA; acceptable: $<0.08$; excellent: <0.05) [28].

\section{RESULTS}

\section{Characteristics of Study Population}

Among the 1,549 OIF/OEF veterans who received the brief TBI screen, 89.6 percent were male $(n=1,388)$ and the mean age was $30.20 \pm 8.39 \mathrm{yr}$ (mean \pm standard deviation). The majority of veterans were white (42.3\%). Most veterans were Active Duty (65.1\%), of Army service (50.5\%), and enlisted (92.3\%) (Table 1).

\section{Demographic and Military Service Comparisons}

Of the 1,549 veterans who were screened, 25 percent $(n=387)$ were TBI+. A comparison of TBI+ and TBIveterans showed that $\mathrm{TBI}+$ veterans were more likely to be male $\left(\chi^{2}=18.3\right.$, degree of freedom $[d f]=1, p<$ $0.001)$, in the Army $\left(\chi^{2}=51, d f=4, p<0.001\right)$, and enlisted $\left(\chi^{2}=13.6, d f=1, p<0.001\right)$ compared with TBI- veterans (Table 2).

\section{Prevalence of Traumatic Brain Injury and Mental Health Screens}

A TBI+ result was associated with a positive PTSD screen $\left(\chi^{2}=188.4, d f=1, p<0.001\right)$ and depression screen $\left(\chi^{2}=94.6, d f=1, p<0.001\right)$ result. Among those who received both mental health screens within 12 months of their TBI screen $(n=1,359), 58$ percent of the TBI+ veterans screened positive for at least PTSD or depression $\left(\chi^{2}=133.9, d f=1, p<0.001\right)$, with 21.3 percent screening positive for both PTSD and depression $\left(\chi^{2}=\right.$ 37.9, $d f=1, p<0.001)$.

When we examined the adjusted relative risks of positive PTSD and depression screens among TBI+ and TBIveterans, TBI+ veterans were 2.1 times more likely to screen positive for PTSD, 2.2 times more likely to screen positive for depression, 1.7 times more likely to screen positive for both PTSD and depression, and 2.2 times more likely to screen positive for either PTSD and/or depression compared with TBI- veterans (Table 2).

\section{Relationships Among Traumatic Brain Injury, Posttraumatic Stress Disorder, and Depression Screen-Based Symptoms}

A four-factor solution emerged from an EFA considering all 12 symptoms included in the TBI, PTSD, and depression screens and allowing the maximum number of factors permitted by the data (7). The fit indices, lack of negative residual variances, and theory all provided support for a four-factor solution. Table 3 presents a summary of the EFA results, including factor loadings (i.e., probit regression coefficients) for the individual items, eigenvalues, and factor correlations. Based on these results, the first factor consisted of five TBI symptoms (excluding sleep problems), the second factor was comprised of the two depression symptoms, the third factor was comprised of the four PTSD symptoms, and a fourth factor consisted of hypervigilance and sleep problems.

Based on the results of the EFA, we specified a model in which five of the six symptoms on the TBI screen loaded onto the latent variable TBI, the two symptoms on the depression screen loaded onto the latent variable depression and the four symptoms on the PTSD screen loaded onto the latent variable PTSD; for the fourth factor, a correlated error was specified between hypervigilance and sleep problems (i.e., the three other factors did not account for the correlation between these two items). The fit statistics from this measurement model indicated a fairly poor fit $\left(\chi^{2}=165.32, d f=26, p<\right.$ 
Table 1.

Demographic characteristics of veterans screened for traumatic brain injury (TBI).

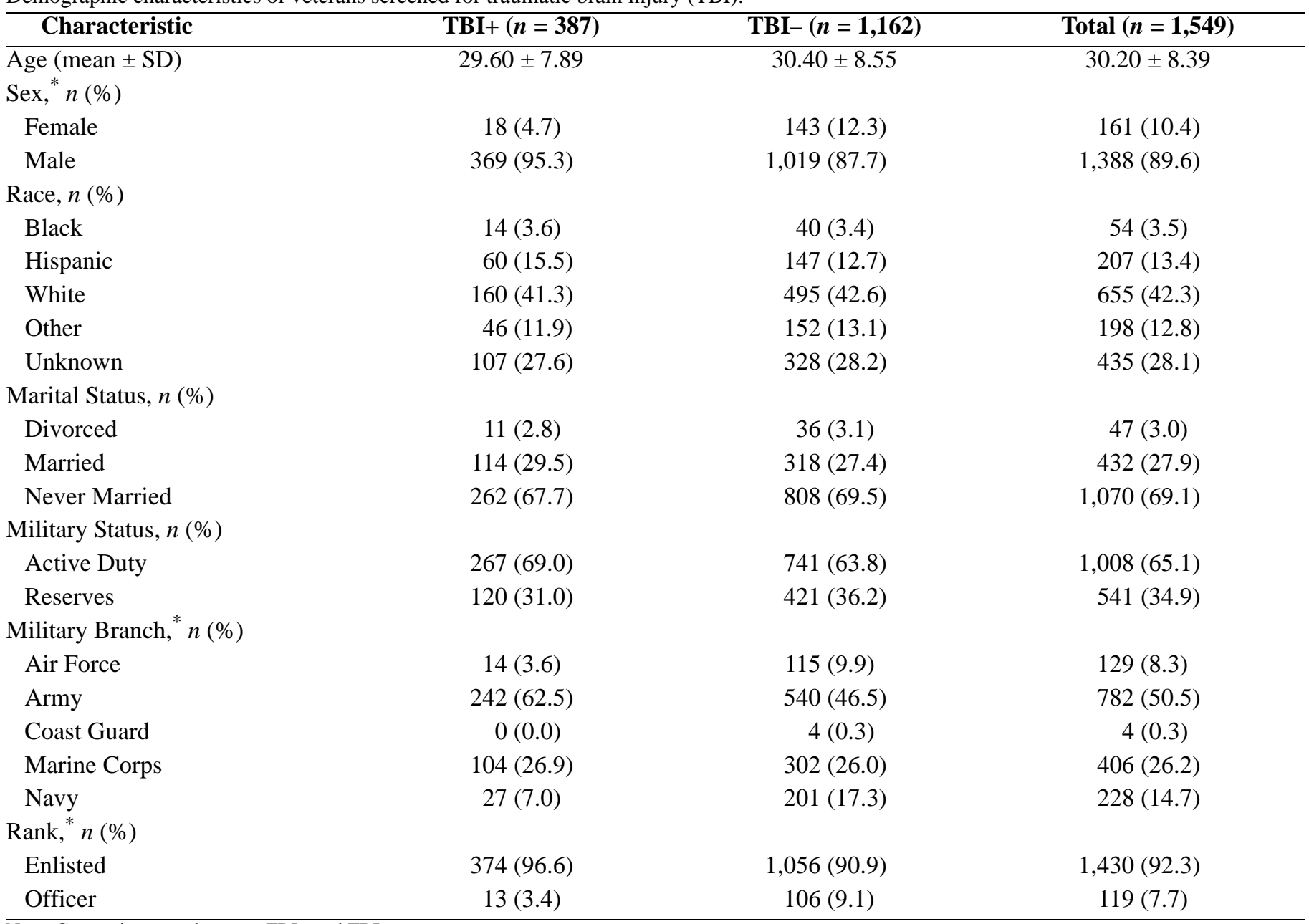

Note: Comparisons are between TBI+ and TBI-.

${ }^{*} p<0.001$.

$\mathrm{SD}=$ standard deviation, $\mathrm{TBI}+=$ positive screen for TBI, TBI- = negative screen for TBI.

Table 2.

Cumulative prevalence rates of positive mental health (MH) screen outcomes among veterans screened for traumatic brain injury (TBI).

\begin{tabular}{|c|c|c|c|c|c|c|c|c|c|}
\hline \multirow{2}{*}{ MH Screen } & \multirow{2}{*}{$\begin{array}{c}\text { Full } \\
\text { Sample }\end{array}$} & \multirow{2}{*}{$\begin{array}{l}\text { TBI+ } \\
n(\%)\end{array}$} & \multirow{2}{*}{$\begin{array}{l}\text { Total } \\
\text { Group }\end{array}$} & \multirow{2}{*}{$\begin{array}{l}\text { TBI- } \\
n(\%)\end{array}$} & \multirow{2}{*}{$\begin{array}{l}\text { Total } \\
\text { Group }\end{array}$} & \multicolumn{2}{|c|}{ Unadjusted Model } & \multicolumn{2}{|c|}{ Adjusted Model $^{*}$} \\
\hline & & & & & & Risk Ratio & 95\% CI & Risk Ratio & $95 \% \mathrm{CI}$ \\
\hline PTSD & $1,400^{\dagger}$ & $254(72.6)$ & 350 & 324 (30.9) & 1,050 & $2.40^{\ddagger}$ & $2.10-2.60$ & $2.10^{\ddagger}$ & $1.87-2.40$ \\
\hline $\begin{array}{l}\text { Depression and } \\
\text { PTSD }\end{array}$ & $1,359^{\S}$ & $112(33.1)$ & 338 & 177 (17.3) & 1,021 & $1.91^{\ddagger}$ & $1.56-2.30$ & $1.74^{\ddagger}$ & $1.40-2.10$ \\
\hline $\begin{array}{l}{ }^{*} \text { Adjusted for age, } \\
{ }^{\dagger} \text { Veterans who rece } \\
{ }^{\ddagger} p<0.001 . \\
{ }^{\S} \text { Veterans who rece } \\
\text { CI = confidence in }\end{array}$ & $\begin{array}{l}\text { marital s } \\
\text { particul } \\
\text { both M } \\
\text { l, PTSD }\end{array}$ & $\begin{array}{l}\text { military stat } \\
\text { screen witl } \\
\text { ens within } 1\end{array}$ & of in & $\begin{array}{l}\text { and rank. } \\
\text { I screen. } \\
\text { reen. }\end{array}$ & . & . & for TBI & & \\
\hline
\end{tabular}


JRRD, Volume 49, Number 7, 2012

Table 3.

Four-factor solution from exploratory factor analysis ${ }^{*}$ for screen-based symptoms of traumatic brain injury, posttraumatic stress disorder, and depression $(n=358)$.

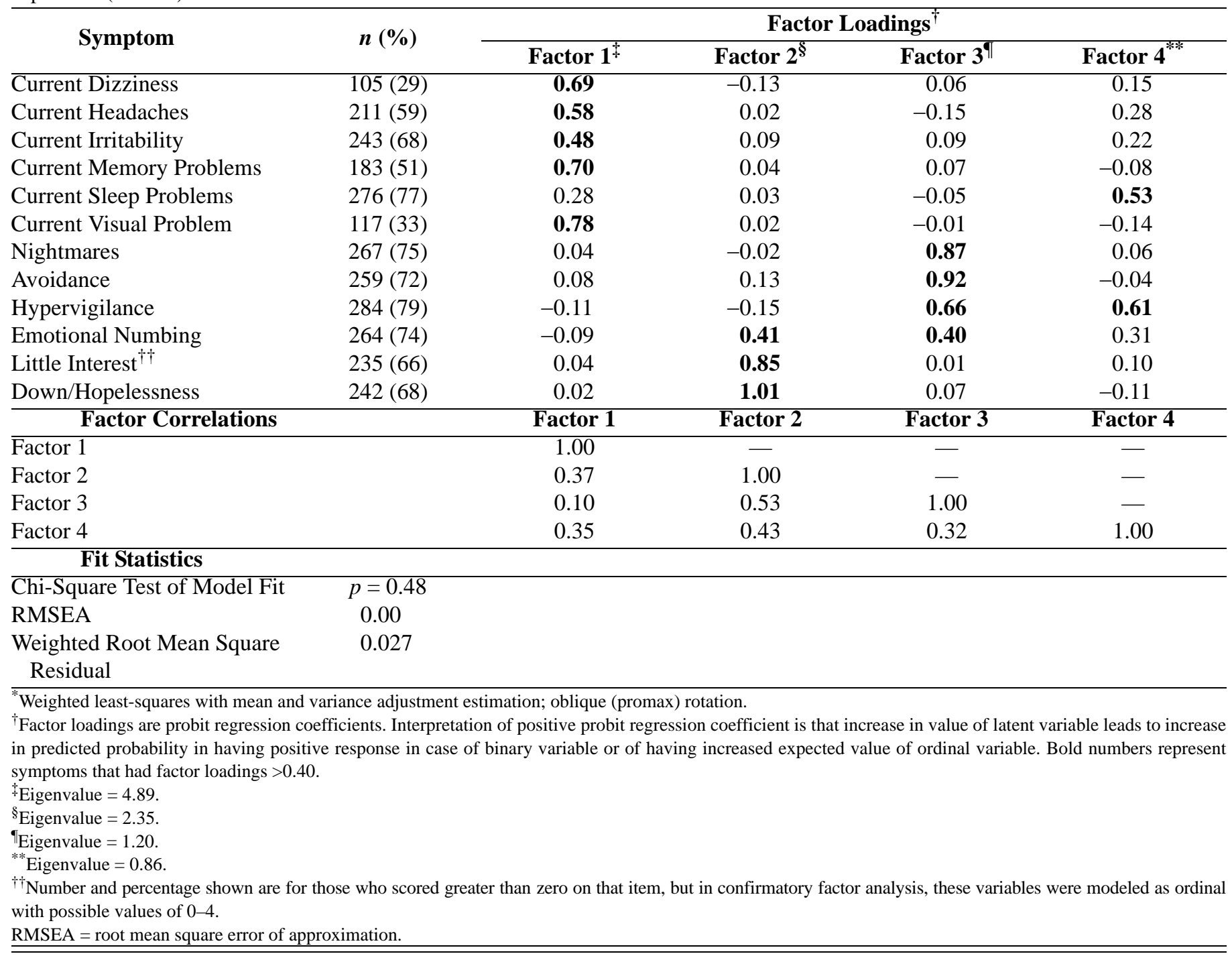

0.001; CFI $=0.952$; TLI $=0.960 ;$ RMSEA $=0.122$ ). Based on a review of the parameter derivatives (modification indices), we made several modifications: (1) sleep was added to the depression factor, (2) sleep was added to the TBI factor, (3) emotional numbing was added to the depression factor, and (4) irritability was cross-loaded onto the PTSD factor. This final model resulted in a favorable chi-square goodness of fit test $\left(\chi^{2}=28.95, d f=\right.$ $27, p=0.36)$; the other fit indices also indicated a very good fit $\left(\chi^{2}\right.$ difference test $=94, d f=4, p<0.001$; CFI $=$ 0.999; TLI = 0.999; RMSEA = 0.014). The Figure shows the final CFA with standardized estimates (probit regression coefficients) and their standard errors. All freely estimated parameters estimates were statistically significant $(p<0.05)$.

The correlation between the TBI factor and the PTSD factor was weak (0.2), indicating that the factor structure distinguished the TBI latent variable from the PTSD latent variable fairly well (Figure). This suggested that there were particular symptoms that were unique to the latent TBI variable (dizziness/balance problems, headache, memory problems, and light sensitivity), while other symptoms (e.g., irritability) overlapped with the PTSD factor. In addition, sleep problems overlapped with the depression factor. Certain symptoms (nightmares, avoidance) were unique to the PTSD factor, but one 


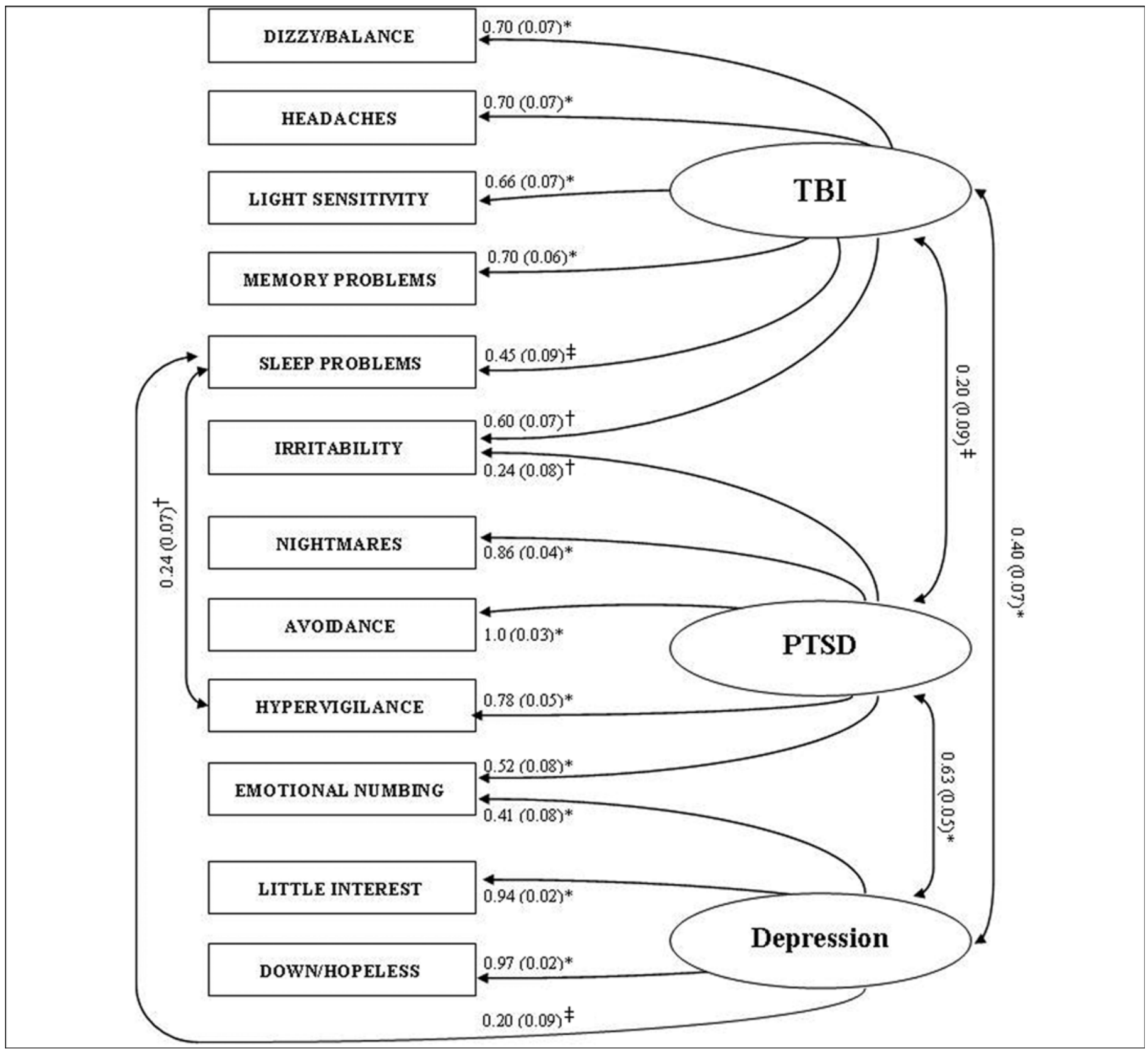

Figure.

Confirmatory factor analysis for symptoms of traumatic brain injury (TBI), posttraumatic stress disorder (PTSD), and depression ( $n=$ 358). Note: Estimates shown are standardized estimates and corresponding robust standard errors. $\chi^{2}=30.076$, degree of freedom $=$ 27, $p=0.31$, Tucker Lewis Index $=0.999$, Comparative Fit Index $=0.999$, root mean square error of approximation $=0.018$. ${ }^{*} p<$ $0.001,{ }^{\dagger} p=0.01,{ }^{\ddagger} p<0.05$.

symptom, emotional numbing, overlapped with the depression factor. We specified the correlated error term between sleep problems and hypervigilance because results from EFA indicated that these symptoms comprised a fourth factor.
$R^{2}$ estimates (squared multiple correlations) provided information on how much variance the factors accounted for in the observed variables. Nightmares and avoidance were well explained by the model $\left(R^{2}=0.74\right.$ and 0.99 , respectively), as were the symptoms of little interest and 
feeling depressed ( $R^{2}=0.87$ and 0.94 , respectively). However, the model did not account well for the six TBIrelated symptoms (all $R^{2}<0.50$ ), particularly sleep problems $\left(R^{2}=0.31\right)$. This suggested that the variability in the distribution of the TBI-related symptoms was not readily explained by co-occurring symptoms of PTSD or depression. In particular, these results supported the idea that sleep problems, the most prevalent TBI-related symptom, were not strongly associated with any of the factors but were still commonly experienced.

\section{DISCUSSION}

Military personnel are returning home with multiple postdeployment conditions and identifying distinguishing features of each condition is critical in order to appropriately triage, diagnose, and provide targeted early interventions. We found that while postdeployment screens accurately identified symptoms associated with TBI, PTSD, and depression, hypervigilance and sleep problems likely constituted a fourth factor that may have represented common postdeployment symptoms due to the high frequency with which these symptoms occurred (79\% and $77 \%$, respectively). Furthermore, we found that while some screen-based symptoms were shared (e.g., irritability), others were unique (e.g., nightmares).

While the TBI screen assessed six symptoms after a TBI injury mechanism had been established, we found that only dizziness/balance problems, light sensitivity, headaches, and memory problems uniquely identified a veteran with mild TBI-related symptoms. In contrast, endorsement of the two other symptoms (sleep problems and irritability) may have led to an inflated false positive rate on the TBI screen because they overlapped with mental health symptoms of PTSD and depression. Similarly, while nightmares and avoidance best captured unique PTSD screen symptoms, hypervigilance and sleep problems constituted common co-occurring postdeployment symptoms. Emotional numbing is an overlapping screen-based symptom shared by PTSD and depression.

These findings suggest that if clinicians want to initially assess for TBI and minimize false positives, focusing on distinguishing rather than shared TBI screen symptoms will be most informative. As always, referral for second-level TBI evaluation by a neurologist or rehabilitation medicine specialist will help determine a veteran's ultimate diagnosis.
Although these distinguishing features suggest possible ways for these screens to be more specific when administered together (i.e., by focusing on distinguishing features of each screen), it will also be important to ensure that doing so will not significantly reduce sensitivity. This is an important area for future investigation.

These findings also have implications for treatment planning. Often, mental health, neurology, and rehabilitation teams must reach consensus concerning the best course of treatment for a particular veteran, and little empirical evidence exists for how to best treat veterans with multiple co-occurring postdeployment symptoms. One possibility is to shift from a diagnosis-based to more of a symptom-based approach, with a particular emphasis on those symptoms associated with functional impairment and problems with reintegration. Our findings suggest that among the most prevalent postdeployment conditions, there are common screen symptoms that may represent shared pathways that can be treated collaboratively across disciplines. For example, there are excellent treatments for irritability and anger and a growing understanding of how to best tailor treatments to target particular types of anger [29]. Given that irritability and anger are shared symptoms of TBI and PTSD, focusing on a symptom-based approach to treatment may help ameliorate both conditions. In addition, there should be more research on the efficacy of treatments that focus on shared symptomatology. For example, treatments that focus on shared symptoms of insomnia, emotional regulation, and cognitive dysfunction may be the best firstline treatments. This shift is consistent with other findings that have stressed the need for integrated care and have highlighted the clinical challenges of addressing PTSD and TBI as separate conditions [17].

Some may argue that symptoms within a common cluster should be treated together as a whole. Indeed, in cases where there is a strong evidence-base for doing so, this is another option: to consider similar symptoms together, such as the distinguishing symptoms of PTSD. These can be targeted through evidence-based treatments (EBTs) such as prolonged exposure and cognitive processing therapy. The same is true for the distinguishing features of depression; EBTs for depression have received excellent support and should be used when these are the presenting symptoms. Once these treatments are administered, symptoms should be re-evaluated and any residual symptoms treated accordingly. Whether a symptom-based or diagnosis-based mental health specialty 
approach is taken also largely depends on contextual factors such as patient preferences, provider training, and systemic resources.

Several limitations should be noted. We used population-based screen data and, as a result, these data should not be generalized to veterans with confirmed diagnoses of TBI, PTSD, and depression. Future investigations should be conducted to better understand whether these findings based on screen-derived symptoms can be replicated in those who receive TBI, PTSD, and depression diagnoses. It is also important to note that these screens are based on self-report rather than direct clinician observation and/or interview or standardized measurement of posttraumatic amnesia and loss of consciousness. Selfreport may also result in the over- or under-estimation of specific symptoms. For example, it may be that because these results are based on self-reported screens, we may have false negatives caused by underreporting or minimizing of symptoms that are consequently not included in these data. Additionally, endorsed symptoms may vary from person to person (e.g., many different types of headaches, with some more severe and disabling) and do not yield any information about severity or intensity (e.g., insomnia once a week vs daily). Screens also may lack sensitivity to detect the level of depression that may include other associated symptoms, such as sleep and memory problems. Because of the way in which the VA TBI screen is structured, those who are TBI- do not complete the entire TBI screen. Consequently, when conducting factor analysis, we could only include veterans who had completed the TBI screen in its entirety (those who screened out did not have the opportunity to respond to the question about current TBI symptoms). Although all screens were done within 1 year of each other, not all were necessarily done at the same time, and in some cases, these screens were conducted several years after the veteran returned home. Despite these limitations, VA screen results represent the level of information that clinicians use to make initial decisions about assessment, triage, and early symptom management of their patients.

\section{CONCLUSIONS}

Overall, we found that TBI+ veterans were approximately twice as likely to also screen positive for depression and PTSD. Four distinct constructs emerged: TBI, PTSD, depression, and a fourth construct consisting of hypervigilance and sleep problems. Screen symptoms unique to TBI include dizziness, headaches, memory problems, and light sensitivity. We found an overlapping screen-based symptom between TBI and PTSD (irritability) and between PTSD and depression (emotional numbing). A symptom-focused, integrated care approach will help to promote more expedient and efficient care for our returning veterans.

\section{ACKNOWLEDGMENTS}

\section{Author Contributions:}

Study concept and design: S. Maguen, K. M. Lau, E. Madden, K. Seal. Acquisition of data: S. Maguen, E. Madden, K. Seal.

Analysis and interpretation of data: S. Maguen, K. M. Lau, E. Madden, K. Seal.

Drafting of manuscript: S. Maguen, K. M. Lau, E. Madden. Critical revision of manuscript for important intellectual content: S. Maguen, K. M. Lau, E. Madden, K. Seal.

Statistical analysis: S. Maguen, E. Madden, K. Seal.

Obtained funding: S. Maguen.

Technical support: K. M. Lau, E. Madden.

Study supervision: S. Maguen.

Financial Contributions: The authors have declared that no competing interests exist.

Funding/Support: This material was based on work supported by the Department of Defense Psychological Health and Traumatic Brain Injury Research Program and a VA Health Sciences Research and Development Service Career Development Award to Dr. Maguen.

Additional Contributions: We thank Gary Abrams, MD; Tatjana Agopian-Novokovic, PhD; Anthony Chen, MD; Don Donati; Charles Filanosky, PhD; Thomas Metzler, MA; Thomas Neylan, MD; and Johannes Rothlind, $\mathrm{PhD}$, for their contributions. Ms. Lau is now the Jesse Brown VA Medical Center, Chicago, Illinois.

Institutional Review: This study was approved by the Committee on Human Research, University of California, San Francisco, and the Human Research Protection Program, San Francisco VA Medical Center.

\section{REFERENCES}

1. Okie S. Traumatic brain injury in the war zone. $\mathrm{N}$ Engl $\mathrm{J}$ Med. 2005;352(20):2043-47. [PMID:15901856] http://dx.doi.org/10.1056/NEJMp058102

2. Terrio H, Brenner LA, Ivins BJ, Cho JM, Helmick K, Schwab K, Scally K, Bretthauer R, Warden D. Traumatic brain injury screening: preliminary findings in a US Army brigade combat team. J Head Trauma Rehabil. 2009;24(1): 14-23. [PMID:19158592] http://dx.doi.org/10.1097/HTR.0b013e31819581d8 
3. Hayward P. Traumatic brain injury: the signature of modern conflicts. Lancet Neurol. 2008;7(3):200-201. [PMID:18275921] http://dx.doi.org/10.1016/S1474-4422(08)70032-2

4. Fischer H. U.S. military casualty statistics: Operation New Dawn, Operation Iraqi Freedom, and Operation Enduring Freedom. RS22452. Washington (DC): Congressional Research Service; 2010.

5. Hoge CW, Goldberg HM, Castro CA. Care of war veterans with mild traumatic brain injury-flawed perspectives. N Engl J Med. 2009;360(16):1588-91. [PMID:19369664] http://dx.doi.org/10.1056/NEJMp0810606

6. Belanger HG, Uomoto JM, Vanderploeg RD. The Veterans Health Administration's (VHA's) Polytrauma System of Care for mild traumatic brain injury: costs, benefits, and controversies. J Head Trauma Rehabil. 2009;24(1):4-13. [PMID:19158591] http://dx.doi.org/10.1097/HTR.0b013e3181957032

7. Veterans Health Administration. Screening and evaluation of possible traumatic brain injury in Operation Enduring Freedom (OEF) and Operation Iraqi Freedom (OIF) veterans. VHA Directive 2007-013. Washington (DC): Department of Veterans Affairs; 2007.

8. Veterans Health Administration. Screening and evaluation of possible traumatic brain injury in Operation Enduring Freedom (OEF) and Operation Iraqi Freedom (OIF) veterans. VHA Directive 2010-012. Washington (DC): Department of Veterans Affairs; 2010.

9. Kim E, Lauterbach EC, Reeve A, Arciniegas DB, Coburn KL, Mendez MF, Rummans TA, Coffey EC; ANPA Committee on Research. Neuropsychiatric complications of traumatic brain injury: a critical review of the literature (a report by the ANPA Committee on Research). J Neuropsychiatry Clin Neurosci. 2007;19(2):106-27. [PMID:17431056]

10. Stein MB, McAllister TW. Exploring the convergence of posttraumatic stress disorder and mild traumatic brain injury. Am J Psychiatry. 2009;166(7):768-76. [PMID:19448186] http://dx.doi.org/10.1176/appi.ajp.2009.08101604

11. Vasterling JJ, Verfaellie M, Sullivan KD. Mild traumatic brain injury and posttraumatic stress disorder in returning veterans: perspectives from cognitive neuroscience. Clin Psychol Rev. 2009;29(8):674-84. [PMID:19744760] http://dx.doi.org/10.1016/j.cpr.2009.08.004

12. Hoge CW, McGurk D, Thomas JL, Cox AL, Engel CC, Castro CA. Mild traumatic brain injury in U.S. soldiers returning from Iraq. N Engl J Med. 2008;358(5):453-63. [PMID:18234750] http://dx.doi.org/10.1056/NEJMoa072972

13. Schell TL, Marshall GN. Survey of individuals previously deployed for OEF/OIF. In: Tanielian TL, Jaycox LH, edi- tors. Invisible wounds of war: Summary and recommendations for addressing psychological and cognitive injuries. Santa Monica (CA): RAND Center for Military Health Policy Research; 2008. p. 87-116.

14. Lew HL, Otis JD, Tun C, Kerns RD, Clark ME, Cifu DX. Prevalence of chronic pain, posttraumatic stress disorder, and persistent postconcussive symptoms in OIF/OEF veterans: polytrauma clinical triad. J Rehabil Res Dev. 2009;46(6):697-702. [PMID:20104399] http://dx.doi.org/10.1682/JRRD.2009.01.0006

15. Schneiderman AI, Braver ER, Kang HK. Understanding sequelae of injury mechanisms and mild traumatic brain injury incurred during the conflicts in Iraq and Afghanistan: persistent postconcussive symptoms and posttraumatic stress disorder. Am J Epidemiol. 2008;167(12):1446-52. [PMID:18424429] http://dx.doi.org/10.1093/aje/kwn068

16. Vanderploeg RD, Belanger HG, Curtiss G. Mild traumatic brain injury and posttraumatic stress disorder and their associations with health symptoms. Arch Phys Med Rehabil. 2009;90(7):1084-93. [PMID:19577020]

http://dx.doi.org/10.1016/j.apmr.2009.01.023

17. Sayer NA, Rettmann NA, Carlson KF, Bernardy N, Sigford BJ, Hamblen JL, Friedman MJ. Veterans with history of mild traumatic brain injury and posttraumatic stress disorder: challenges from provider perspective. J Rehabil Res Dev. 2009;46(6):703-16. [PMID:20104400] http://dx.doi.org/10.1682/JRRD.2009.01.0008

18. Manpower Data Center Contingency Tracking System Deployment File. OEF/OIF roster. Washington (DC): Department of Defense; 2009.

19. Schwab KA, Ivins B, Cramer G, Johnson W, Sluss-Tiller M, Kiley K, Lux W, Warden D. Screening for traumatic brain injury in troops returning from deployment in Afghanistan and Iraq: initial investigation of the usefulness of a short screening tool for traumatic brain injury. J Head Trauma Rehabil. 2007;22(6):377-89. [PMID:18025970] http://dx.doi.org/10.1097/01.HTR.0000300233.98242.87

20. U.S. Government Accountability Office. VA health care: Mild traumatic brain injury screening and evaluation implemented for OEF/OIF veterans, but challenges remain. GAO-08-276. Washington (DC): U.S. Government Accountability Office; 2008.

21. Prins A, Ouimette $\mathrm{P}$, Kimerling $\mathrm{R}$, Cameron RP, Hugelshofer DS, Shaw-Hegwer J, Thrailkill A, Gusman FD, Sheikh JI. The Primary Care PTSD screen (PC-PTSD): Development and operating characteristics. Prim Care Psychiatry. 2004;9(1):9-14.

22. Prins A, Ouimette $\mathrm{P}$, Kimerling $\mathrm{R}$, Cameron RP, Hugelshofer DS, Shaw-Hegwer J, Thrailkill A, Gusman FD, Sheikh JI. The Primary Care PTSD screen (PCPTSD): Corrigendum. Prim Care Psychiatry. 2004;9:151. 
23. Calhoun PS, McDonald SD, Guerra VS, Eggleston AM, Beckham JC, Straits-Troster K; VA Mid-Atlantic MIRECC OEF/OIF Registry Workgroup. Clinical utility of the Primary Care-PTSD Screen among U.S. veterans who served since September 11, 2001. Psychiatry Res. 2010; 178(2):330-35. [PMID:20483463] http://dx.doi.org/10.1016/j.psychres.2009.11.009

24. Kroenke K, Spitzer RL, Williams JB. The Patient Health Questionnaire-2: validity of a two-item depression screener. Med Care. 2003;41(11):1284-92.

[PMID:14583691] http://dx.doi.org/10.1097/01.MLR.0000093487.78664.3C

25. Kroenke K, Spitzer RL, Williams JB. The PHQ-9: validity of a brief depression severity measure. J Gen Intern Med. 2001;16(9):606-13. [PMID:11556941] http://dx.doi.org/10.1046/j.1525-1497.2001.016009606.x

26. Tucker LR, Lewis C. A reliability coefficient for maximum likelihood factor analysis. Psychometrika. 1973;38(1):1-10. http://dx.doi.org/10.1007/BF02291170

27. Bentler PM. Comparative fit indexes in structural models. Psychol Bull. 1990;107(2):238-46. [PMID:2320703] http://dx.doi.org/10.1037/0033-2909.107.2.238

28. Browne MW, Cudeck R. Alternative ways of assessing model fit. In: Bollen KA. Long JS, editors. Testing struc- tural equation models. Newbury Park (CA): Sage Publications; 1993. p. 136.

29. Elbogen EB, Wagner HR, Fuller SR, Calhoun PS, Kinneer PM, Beckham JC; Mid-Atlantic Mental Illness Research, Education, and Clinical Center Workgroup. Correlates of anger and hostility in Iraq and Afghanistan war veterans. Am J Psychiatry. 2010;167(9):1051-58. [PMID:20551162] http://dx.doi.org/10.1176/appi.ajp.2010.09050739

Submitted for publication February 9, 2011. Accepted in revised form June 17, 2011.

This article and any supplementary material should be cited as follows:

Maguen S, Lau KM, Madden E, Seal K. Relationship of screen-based symptoms for mild traumatic brain injury and mental health problems in Iraq and Afghanistan veterans: Distinct or overlapping symptoms? J Rehabil Res Dev. 2012;49(7):1115-26.

http://dx.doi.org/10.1682/JRRD.2011.02.0015

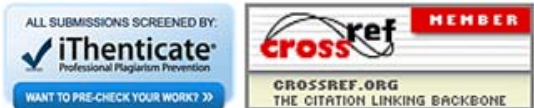


